\title{
Quantification of Uncertainty for Numerical Simulations with Confidence Intervals
}

\author{
James Glimm \\ Distinguished/Leading Professor \\ Dept of Applied Mathematics and Statistics \\ P-138A Math Tower \\ University at Stony Brook \\ Stony Brook, NY 11794-3600 \\ Phone: +1-(516)-632-8355 \\ FAX: +1-(516)-632-8490 \\ glimm@ams. sunysb.edu
}

\begin{abstract}
We present a prediction and uncertainty assessment methodology for numerical simulation. The methodology allows prediction of confidence intervals. It has been developed jointly with a number of colleagues. It is a work in progress in the sense that not all components of the methodology are complete. The methodology, at its present level of deveopment, will be illustrated in two specific cases: the flow of oil in petroleum reservoirs (with prediction of production rates) and an analysis of solution errors for the simulation of shock wave interactions.

The formalism assesses uncertainty and yields confidence intervals associated with its prediction. In the terminology of verification and validation, these predictions can be verified as exact within a framework for statistical inference, but they are not validated as being descriptive of a physical situation. In fact the present illustrative examples are simplified not intended to represent an experimental or engineering system.

The methodology combines new developments in the traditional areas of oil reservoir upscaling and history matching with a new theory for numerical solution errors and with Bayesian inference. For the shock wave simulations, the new result is an error analysis for simple shock wave interactions.

The significance of our methods, in the petroleum reservoir context, is their ability to predict the risk, or uncertainty associated with production rate forecasts, and not just the production rates themselves. The latter feature of this method, which is not standard, is useful for evaluation of decision alternatives. For shock wave interactions, the significance of the methodology will be to contribute to verification and validation of simulation codes.
\end{abstract}

V.N. Alexandrov et al. (Eds.): ICCS 2001, LNCS 2073, p. 5, 2001.

(C) Springer-Verlag Berlin Heidelberg 2001 